\title{
La gerencia pública en la sociedad post-industrial
}

\section{Guerrero, Omar*}

\section{Resumen}

Hoy en día la sociedad postindustrial se manifiesta en cinco dimensiones: un sector económico; una distribución ocupacional; la centralidad del conocimiento teórico; una orientación del futuro con base en el control de la tecnología y una hechura de decisiones orientadas por la nueva tecnología intelectual. De ahí nace la motivación del presente trabajo, el cual consiste en analizar la gerencia pública en la sociedad postindustrial, a través del análisis documental. Así, en consonancia con las condiciones presentes, esta sociedad da cobijo a una administración pública que se consolida en las relaciones de dominación, es decir el gobierno de los hombres; junto a una gerencia que asume la gestión de las cosas. Actualmente, en suma, la administración pública mantiene sus rasgos esenciales, sin menoscabo de convivir una época en la cual los cambios producidos por la post- industrialización provoca el advenimiento de la modalidad d la gerencia de servicios.

Palabras clave: Gerencia pública, sociedad postindustrial, gobierno de los hombres, gerencia de las cosas.

\section{Public Management in Post-Industrial Society}

\begin{abstract}
Post-industrial society manifests itself in five dimensions: an economic sector; an occupational distribution; centralism of theoretical knowledge; a futuristic orientation based on technological control and decision making oriented towards new intellectual technology. This motivates us to analyze public management in the post-industrial society through documentary analysis. According to present conditions, this society gives rise to a public administration that consolidates relations of dominion, that is the government of men together with a management that assumes control of material things. Presently, public administration maintains its basic characteristics, without at the same time taking into account the stage in which changes produced by post-industrialization impel the advent of the modality of management of services.
\end{abstract}

Key words: Public management, post-industrial society, human government, management of materials.

Recibido: 04-07-08. Aceptado: 05-03-21

Investigador de la Universidad Nacional Autónoma de México (UNAM).

E-mail: omarg@servidor.unam.mx 


\section{Introducción}

Cada etapa del desarrollo de la sociedad tiene uno o varios rasgos significativos, que la definen o delinean en sus trazos relevantes. Hoy en día, tiempo en el que el Estado ostenta los rasgos esenciales que le caracterizan hasta el presente -y su administración se personifica en las formas actuales-, su devenir tiende a ser representado por dos modos opuestos. A ello contribuyó determinantemente la emergencia de la industrialización, la cual, abandonando los moldes artesanales -de la misma forma que la mariposa deja el capullo primigenio-, prohijó el advenimiento de una modalidad hasta entonces inédita de gestión llamada "gerencia" (manejo, management) ${ }^{1}$. El locus donde fue gestada: los procesos de producción basados en el uso de la máquina, engendraron paralelamente nuevas relaciones de producción, y por consiguiente, nuevos administradores (gerentes) y nuevos administrados (obreros).

Uno de los rasgos sobresalientes de nuestro tiempo, por lo tanto, es la convivencia de dos modos de gestión paralelos y diversos: la administración y la gerencia; que se tocan rozándose o chocando, produciendo de su contacto una relación más tensa que tersa.

Sin embargo, la economía y su gerencia buscan un sitio junto al Estado y su administración -ambos más antiguos-, creándose relaciones de ajuste que tie- nen a ser ríspidas, principalmente por motivos emanados de los problemas de su mutua incompatibilidad. Lo antiguo y lo nuevo tan luego se ajustan, pronto se desajustan, estableciendo un proceso perpetuo de acomodos y re-acomodos que es visible a través del pensamiento administrativo, tanto el público, como el privado (Saint-Simon, 1975), (Osborne y Gaebler, 1992), (Farol y Gelinier, 1967).

\section{De la sociedad pre-industrial, a la sociedad industrial}

En tanto la administración pública se consolida en torno a las relaciones de dominación, personificando propiamente al gobierno de los hombres, la gerencia asume el carácter de gestión de las cosas. La primera, que en su infancia tuvo un papel gerencial eminente como constructora de obras públicas colosales y como prestadora de servicios públicos titánicos, fue ciñendo sus funciones a su trato con los hombre a través del poder encarnado en el Estado administrativo (Wittfogel, 19579. Esta condición fue el efecto, entre otros, del eclipse del mercantilismo y la consolidación de la economía como una disciplina autónoma. Estando las relaciones de producción apartadas de las relaciones políticas, a medida que los rasgos gerenciales del Estado se atenúan, la gestión de las cosas se convierte en una función puramente eco-

1 La descripción primigenia del surgimiento de la gerencia dentro de los moldes industriales, es narrada por Marx (1968: 366-372) cuando trata de la cooperación y la división del trabajo. El desarrollo más extenso del tema gerencial se debe a Farol $(1931,1927)$, que lo trató profunda y continuamente en varias de sus obras. 
nómica cuyos portadores son los productores privados: los industriales, los empresarios y los gerentes.

El fruto de estos cambios es un escenario social emergente cuyo emblema es una industrialización que va dejando su sello en la sociedad, y su acento en la fabricación de objetos inanimados. Es la industrialización la que ofrece las condiciones de un desarrollo próspero y singular basado en el dominio de la naturaleza, ofreciendo a las relaciones económicas emergentes como sede de un nuevo género de gestión: la gerencia.

Ese género de administración está constituido con materiales diametralmente distintos a la administración del Estado, cuyo origen y asiento son las relaciones políticas, pues aquella otra es el fruto de las relaciones económicas. No es el Estado, sino la industria, el escenario "posible" para una vida superior del ser humano por cuanto que establece un régimen basado en la jerarquía de competencias técnicas para sustentar a la gestión.

\section{De la sociedad industrial, a la sociedad post-industrial}

La sociedad industrial es un concepto incluyente que ha abarcado experiencias, aproximadamente, de una docena de países diferentes y que comprende sistemas políticos de sociedades que antaño fueron antagónicas, como los Estados Unidos y la desaparecida Unión Soviética. La sociedad industrial, que fue organizada sobre la base de la producción y la maquina, pues su objeto es la fabricación de bienes materiales, reemplazó a la sociedad pre-industrial cuyo fundamento descansaba en las fuentes de trabajo natural y la extracción de los recursos primarios que brinda la naturaleza (Bell, 1976: 12 y 30).

Pero la sociedad industrial está cambiando rápidamente, pues desde hace tres décadas atrás ya está siendo sustituida por la sociedad post-industrial, cuyo significado se expresa en cinco dimensiones:

- el sector económico cuyo carácter es el cambio de una economía que produce mercancías, a otra economía que produce servicios;

- la distribución ocupacional, cuya índole es la preeminencia de las clases profesionales y técnicas;

- el principio axial, es decir, la centralidad del conocimiento teórico como fuente de la innovación;

- la orientación hacia el futuro, bajo la forma de control sobre la tecnología;

- y finalmente, la hechura de decisiones como modo de creación de una nueva tecnología intelectual.

Uno de los problemas característicos de la época actual, es decir, la sociedad post-industrial, es el incremento de la ancestral tensión entre el Estado y la economía, que tiende a centrarse en los desafíos gerenciales al sistema político. Debido a que una sociedad consciente de su destino -que busca controlar su propia suerte-, se caracteriza por un orden político que lo abarca todo, tiende a sufrir presiones nacidas de su propio desarrollo. Como en la sociedad industrial, la importancia del factor técnico del conocimiento aumentó aceleradamente. Ello impulsa a sus líderes, es decir: los científicos, los ingenieros y los tecnócratas, a competir con los políticos (Bell, 1976: 29). 
Las formas de vida emergentes, que dependen de la primacía del saber teórico, retan inevitablemente a la cultura dominante. La sociedad post-industrial se plantea el mismo problema que la sociedad industrial en sus albores, es decir, la primacía del conocimiento, pero con la novedad de no tratarse de conocimiento práctico, sino teórico.

\section{La gerencia cataláctica}

Hay que hacer notar que las condiciones sociales narradas, entre cuyas características se encuentra la preeminencia de los servicios, así como la presencia de las clases profesionales y técnicas, ha fortalecido otro rasgo eminente de los tiempos actuales. Se trata de otro forma de gestión emanada del papel del mercado en los procesos económicos.

Ese nuevo escenario inspiró el uso renovado de un antiguo término económico: catalaxia o trueque, es decir: mecanismos de mercado a través de la coordinación; así como el papel del sistema de precios como medio de información entre los participantes. Tal ámbito de intercambio que trasciende el locus de la producción y el proceso fabril, entraña mecanismos espontáneos y automatismos cibernéticos, que de conformidad con el ideario de la economía neoclásica, hacen superflua la gerencia concebida como una organización o un ente corpóreo.

Lo dicho requiere, evidentemente, una explicación.

Hay que partir del hecho de que hasta entonces sólo existían, no dos especies de un género único, sino dos géneros de gestión paralelos, independientes y autónomos, pero vinculados y conti- guos en los temas de su interés común. Son la administración y el management. Junto con ellos ha brotado un tercer género, un ente distinto a lo observado por Charles-Jean Bonnin o por Henri Farol (Bonnin, 2004) es decir, un tercer género de gestión nacido de las relaciones comerciales, una gerencia cataláctica cuyo objeto existencial mira hacia el mercado y se explica desde el mercado.

Gerencia cataláctica es el management cuya operación se realiza con base en un cálculo monetario, que tiene su sede causal en las relaciones de mercantiles. Es aquella gestión donde cada persona cooperante es evaluada en función del valor de su contribución en el proceso de producción de bienes y servicios. Salarios y sueldos no dependen de decisiones arbitrarias, sino de mandatos de los consumidores dictados desde el mercado, lo mismo que las mercancías que brotan de los procesos productivos de la empresa. Es el "consumidor soberano" quien manda sobre los empresarios, los agricultores y los trabajadores, cuyas ganancias, pérdidas y salarios, están determinados por los procesos mercantiles. La fuerza de su mandato no deriva de que involucre a personas relacionadas en el proceso de producción, sino a las cosas que ese proceso implica. La gerencia cataláctica "concierne a las cosas, no a los hombres" (Misses, 1996:38).

Se trata pues de la prolongación y la sublimación de la administración de las cosas anunciada muchos años antes por Henri de Saint-Simon (1975), donde se gestiona sin prejuicio ni pasión, y con la pura objetividad de tratar con materiales inanimados.

El surgimiento de la gerencia cataláctica es explicable a partir de las trans- 
formaciones habidas en la economía moderna, así como en el mercado y la empresa privada.

\section{Las transformaciones en la gerencia privada}

Una fuente confiable de explicación acerca de la emergencia de la gerencia cataláctica, yace en la teoría del negocio lucrativo de la empresa privada moderna. Según las observaciones más recientes, hoy en día los procesos económicos se orientan hacia el comerciante, más que al fabricante, como aquél que conoce mejor al cliente. Al mercader se confía el diseño de los productos y su desarrollo, así como buscar fabricantes que hagan los artículos según sus diseños, especificaciones y costos (Drucker, 1996: 21-22).

Ese cambio propició que la gerencia desplazara su foco de atención desde el tradicional nicho de la producción y lo trasladara hacia el comercio, trascendiendo su ancestral locus en los procesos de fabricación, para transitar hacia los procesos mercantiles.

A partir de entonces la empresa privada dedica buena parte de su tiempo a estudiar lo que ocurre fuera de sí misma, auscultando el sentir y la opinión de los clientes, o más bien de sus no-clientes, pues los primeros síntomas de alteración esencial de una firma raramente emergen dentro de su organización, o entre sus clientes efectivos (Drucker, 1996:30). Hay que enfatizar que, habiendo la gerencia privada trascendido la interioridad de la empresa, los clientes dejaron de ser sus interlocutores, para empeñar su esfuerzo en captar a lo que no lo son todavía. Esos síntomas normalmente se ma- nifiestan primero entre los que no-clientes, que siempre son mucho más numerosos que los clientes.

Peter Drucker fue el primero en observar este fenómeno de transformación de la gerencia privada cuando estudió el caso de la firma británica Marks and Spencer, que fue pionera en diseñar y desarrollar las mercancías con base en la expectativa de compra del cliente potencial, más que en los incentivos de venta con criterios de producción. Un acicate a favor del no-cliente es la ausencia de control sobre el mercado. Drucker ejemplifica con WallMart, un emporio gigantesco del comercio, que a mediados de la década de 1990 detentaba sólo el $14 \%$ del mercado de bienes de consumo en los Estados Unidos, de modo que el $86 \%$ del mercado era no-cliente de esa empresa. La lección explícita es que una empresa lucrativa debe atender preferentemente al mercado, es decir, a los no-clientes, pues sólo de esa forma será efectivamente un negocio productivo.

$\mathrm{Y}$, consiguientemente, para que la administración pública sea eficiente por cuanto a la provisión de bienes y servicios, debe ser rentable; para ser rentable debe orientarse al cliente (y no-cliente, según el caso); y para orientarse el cliente debe evadirse de su nicho burocrático y situarse en el mercado. Debe, en suma, adoptar la neogerencia pública como su mentor y así incorporarse a los progresos de la economía neoclásica globalizada.

$Y$ en eso consiste precisamente el cúmulo de incentivos de la reforma de la administración pública, la cual, en la era de la sociedad post-industrial, está enfrascada en un proceso de cambio y de asimilación de las prédicas provenientes de la nueva gerencia pública. 


\section{Efectos de la irrupción de la nueva gerencia pública en la administración pública}

En la década final del siglo pasado y en los primeros años del actual, las sociedades modernas han sufrido cambios acelerados y radicales, que las han hecho mudar gobiernos, transformar economías y alterar administraciones públicas. Sobre este último punto se debe destacar la irrupción de nuevos modelos de implementación, particularmente la nueva gerencia pública, así como la invasión de un lenguaje gerencial novedoso, y promoción de valores diversos a los hasta ahora vigentes. Asimismo, hay una reivindicación radical de antiguas propuestas, como la eficiencia de desempeño administrativo, y la exaltación de las conductas y procedimientos de organizaciones diversas a la administración pública.

Tal escenario se ha visto influenciado determinantemente por un factor externo: la globalización, que opera internamente en las administraciones nacionales, inyectándolas de inter y supra-nacionalidad. El resultado es una colisión de paradigmas de implementación en administración pública, entre la metodología clásica a través de procedimientos políticos y jurídicos, y otra caracterizada por mecanismos económicos de mercado y competencia. Paradójicamente, esta confrontación no ha producido un debilitamiento del primer paradigma en provecho del segundo, o su reemplazo, sino una convivencia donde el modo clásico de implementar ha prevalecido.

Sucedáneamente, la incorporación de procesos de mercado en la producción de bienes y la provisión de los servicios públicos ha estado limitada por normas de regulación necesarias, además de inevitables, que patentizan que aquélla convivencia señalada se reproduce en la coexistencia y colaboración del Estado y el mercado.

A pesar del influjo de los cambios señalados, formas, usos y procedimientos añejos no sólo se perpetúan, sino se afianzan y se expanden, quizá motivados por el riesgo que entraña la transformación. Esto explica por qué, junto con los afanes integracionistas, las nacionalidades tienden a fortalecerse con base en aquello que Siegfried (1965) llamó el alma de los pueblos. Consiguientemente, existen pueblos que están viviendo en la dicotomía de ser nacionales en su país y ciudadanos comunitarios en Europa (Siegfried, 1995).

Naturalmente la situación narrada tiene reflejos muy intensos en el estudio de la administración pública, así como de su naturaleza, objeto, efectos y relaciones. Hay que recordar que la ciencia de la administración pública siempre ha tenido una relación tensa con otras disciplinas hermanas, principalmente con la economía y el derecho, así como la gerencia privada. Esta situación ha determinado una tendencia manifiesta a fortalecer su centro conceptual, donde se halla el Estado, y delinear fronteras flexibles para que funcionen como aduanas por donde penetra el conocimiento de otras disciplinas. Empero, estas fronteras han tendido a ser trincheras de confrontación conceptual, o tierra de nadie, provocando injustificadas incertidumbres sobre los límites de la administración pública como ciencia.

Otro tanto ocurre con su arte, es decir, con su ejercicio profesional diferenciado, que con alguna frecuencia se cree 
igual al propio de los gerentes privados. El acecho a los servicios civiles de carrera, o su desmantelamiento, han contribuido a desfigurar el tipo de desempeño del administrador público, al enturbiar su ámbito laboral, desdibujar los programas de estudio con los cuales se forman, y soslayar los títulos y grados que avalan su desempeño.

Finalmente hay que considerar a la administración pública como institución, es decir, como un conjunto de prácticas, normas y organizaciones que permanecen en el tiempo, y dan identidad al quehacer de quienes tienen a su cargo la gestión de los asuntos públicos. Tal como es observable en las dependencias que forman la administración pública, salvo los países de la antigua órbita socialista de Europa, todos los demás poco han cambiado la fisonomía de sus organizaciones, a pesar de las transformaciones narradas. Hay que añadir, que aquellos países ex-socialistas están mudando hacia las formas propias de sus vecinos occidentales.

En fin: persiste el antiguo antagonismo que modela el curso de la humanidad, entre lo que permanece y lo que cambia.

\section{El temario actual}

Lo antes narrado invita a la exposición de seis temas claves sobre el desarrollo de la administración pública contemporánea. Los cuales, como bien lo determinó Hinzte (1968: 298), parafraseándolo, conciben a la administración pública no como algo que es de sí para siempre, sino que deviene y se transforma para seguir esencialmente siendo lo que es.

\subsection{Definición de la Administración Pública}

Siendo la administración pública inherentemente la administración del Estado, por principio, se define necesariamente con base en el uso de la fuerza legítima -que es el principio del Estado. La introducción de los mecanismos de mercado, que son horizontales y no-coercitivos, de ser operantes y efectivos, demandarían necesariamente un matiz dentro de dicha definición.

A la administración pública no le es posible escapar de su naturaleza política, porque de hacerlo, dejaría de ser administración pública. Llenado con economía el vacío dejado por la política, se convertiría en gerencia; dejando de ser política, perdería su pública denominación para asumir la gestión de lo privado. Se habría cumplido la profecía de Henri de Saint-Simon, en el sentido de que habrá llegado por fin el tiempo en el cual impera la administración de las cosas de las tenemos necesidad, ya no del gobierno de los hombres (Saint Simon citado por Gide 1913: 109).

No está de más recordar que "la administración pública es la que tiene la gestión de los asuntos comunes respecto de la persona, de los bienes y de las acciones del ciudadano como miembro del Estado, y de su persona, sus bienes y sus acciones como incumbiendo al orden público" (Bonnin, 2004:229).

\subsection{La Administración del Estado}

Siendo la administración pública inherentemente la administración del Estado, y no pudiendo ser otra cosa en tanto 
el Estado exista, su definición debe plantear (y/o replantear) el aspecto administrativo del Estado. Es decir, lo administrativo como lo propio de la administración pública como ciencia, así como lo económico lo es de la ciencia económica y lo político de la ciencia política.

No debemos olvidar que Dwight Waldo explicó magistralmente, que si en 1948 usó el pleonasmo Estado administrativo para titular una de sus grandes obras (Waldo, 1948), ello obedece a que los estados son administrativos o no son estados (Waldo, 1990:79).

El Estado administrativo no ha desaparecido de la escena, sino que se ha transformado.

Este cambio es observable en una tesis que sostiene una mutación asombrosa del Estado administrativo, en el "Estado no-administrativo". La idea es de Durant (1998: 211-212), quien declara que en los Estados Unidos es perceptible ese cambio desde las décadas de 1980 y 1990, y que una tercera ola -parafraseando a Alvin Toffler- está hoy en día reemplazando a aquellas dos que hicieron pasar a ese país de una sociedad rural a una sociedad industrial. Esa tercera ola, plasmada en el capitalismo global, está representada por la transformación del Estado administrativo en un "Estado no-administrativo", que tiene nuevos objetivos, alcances y complejidades que afrontar. La causa es que los esfuerzos por reducir la "magnitud" del Estado administrativo redundaron en una disminución de burócratas, pero no de burocracias ahora laborando en las redes organizativas del público, de las empresas privadas y de las entidades no-lucrativas, que asumieron las funciones antaño a su cargo. La burocracia, sencillamente, cambió de lugar.

Igualmente, dependiendo de nuevos esquemas asociativos (conocidos como actores no-gubernamentales) los programas gubernamentales implementados se han extendido más allá de lo que estaban en la época del Estado administrativo. Este panorama, que muestra el ascenso del "Estado no-administrativo", cuyo poder lo ejerce en nombre de la sociedad, está pasando de la esfera pública hacia el mercado y las esferas cívicas de la nación.

El "Estado no-administrativo" así concebido por Durant resulta tener menos administración que el Estado administrativo, pero igual o más burocracia, así como mayor rango de complejidad y magnitud de actividades programáticas. La paradoja del "Estado no-administrativo" es que, negando su parentesco con el Estado administrativo, termina por asumir sin medida alguna la esencia de aquél: la burocracia.

\subsection{La Administración de lo Público}

Siendo la administración pública inherentemente la administración del Estado, es propiamente pública. Hay que hacer notar que el Estado representa, esencialmente, el aspecto público de la convivencia humana, pues a través del derecho público que se norma la convivencia de una nación, así como la garantía del disfrute de los espacios públicos para todos. Sin embargo, existe una diversidad de espacios públicos que no se limitan al Estado, sino más bien, son propios de la convivencia social y entrañan al 
concepto de ciudadano. Es la ciudadanía, consiguientemente, la que define in extenso lo público de la administración.

Así, bajo la idea de un Estado administrativo que ha alcanzado hasta ahora una vida perpetua gracias a su transformación, lo público de su administración se visualiza con énfasis, por cuanto esa administración es concebida como la implementación de policy por los regímenes en pro de ese público integrado por seres humanos. La política opera como la sangre vital de la administración, de modo que sus relaciones son explicables a partir de un complejo vinculatorio cuya sede es la civilización occidental (Gawthrop, 1998:2). Los imperativos humanísticos se convierten en los criterios centrales de la administración pública, pues ella debe operar dentro del mandato de los valores democráticos de la libertad, la igualdad, la justicia y la responsabilidad.

En todo caso, la administración pública es más un gobierno de los hombres, que una administración de las cosas.

\subsection{La Gerencia de la Administración Pública}

La política ocupa un lugar prioritario en el estudio de la administración pública contemporánea, pero esto no significa que se olvide la gerencia. Henry (1990: 3-26) utiliza una figura muy representativa en la cual la administración pública aparece acompañada por dos familiares muy cercanos: una madre natural, la ciencia política, y un padre adoptivo: el manejo; de modo que su desarrollo está influenciado por ambos progenitores.

La gerencia de bienes inanimados ha sido un tema perpetuo entre los pen- sadores administrativos privados. Esta visión está firmemente arraigada, como es patente en el ideario de Sheldon (1986:35), donde esa gerencia es exaltada con un vigor extraordinario. De hecho, es la base de la gerencia privada por cuanto ciencia.

Sheldon (1986) -de manera similar a Saint-Simon- sostuvo, que siendo la ciencia apta para elaborar principios de la planificación del trabajo susceptibles de aplicación universal, inevitablemente debe considerar la reserva de que están sujetos a los caprichos del factor humano. Es decir, que, "en otras palabras, en la medida en que el management trata de cosas, sus métodos pueden reducirse a términos de principios científicos, pero en la medida en que trata con seres humanos, únicamente puede utilizar principios científicos en la medida en que los seres humanos estén dispuestos a sujetarse a ellos" (Sheldon, 1986:35). La gerencia está más cómoda, pues, manejando cosas, que tratando con hombres, de modo que la "ciencia del management" deriva en conclusiones relativas a cada uno de los elementos que intervienen en el trabajo del hombre, pero es solamente el arte del managerlo inducirá a poner esas conclusiones en práctica. Esto obedece a que, si bien, existe una ciencia de la contabilidad de costos, planificación, fabricación y comunicación, la ciencia de la cooperación humana es existente.

Sin embargo, la gerencia de bienes inanimados no puede prescindir de las relaciones sociales, las cuales persisten a pesar de estar separadas del imperio del poder y el dominio (gobierno de los hombres), pues ellas se sitúan en el ámbito al que Marx (1968:38) se refirió como feti- 
chismo de la mercancía. Es decir, los vínculos comunes entre los seres humanos se han consificado al punto que ahora existen relaciones materiales entre personas y relaciones sociales entre cosas.

La administración pública no puede prescindir de su índole antropocrática, ni de su carácter fisiocrático. Esto es patente en el pensamiento de uno de los próceres intelectuales estadounidenses, Leonard White, quien siendo un lector cuidadoso de Sheldon, no dejó de percibir el asunto de un modo diverso. White definió a la administración pública como el manejo de hombres y materiales para lograr los propósitos del Estado (White, 1926:2). White se propuso estudiar la organización, el personal, las finanzas y el control, que constituyen la división original de la gerencia en la administración pública.

De manera casi idéntica a White, Dwight Waldo la definió como la organización y el manejo de hombres y materiales para lograr los propósitos del gobierno (Waldo, 1967). Está claro en ambos escritores la idea de no prescindir del gobierno de los hombres ni de la administración de las cosas, pues del mismo modo como la administración pública emana del gobierno, tiene inherentemente su gerencia de bienes inanimados.

Hay pues una gerencia inherente a la administración pública, propia, y diversa a la gerencia de producción y a la gerencia cataláctica.

Esto no escapó al fundador de nuestra disciplina, quien en una definición temprana de la administración pública, la concibió como "una potencia que arregla, corrige y mejora cuanto existe, y da una dirección más conveniente a los seres organizados y a las cosas" (Bonnin, 2004:265).

\subsection{Globalización: el Factor Mercado}

La globalización aporta el factor mercado, poniendo el énfasis en la economía, y por consiguiente, en la organización del Estado en las materias hacendaria, industrial, agrícola, comercial y monetaria. Los cambios en la administración pública, aquí, se perciben en las organizaciones que se vinculan con la materia económica en general. Naturalmente, también aquí, es donde se percibe con mayor fuerza la reforma neogerencial.

Uno de los rasgos más significativos de la economía neoclásica consiste en su fe en el mercado. Pero, significativamente, los economistas que militan en esa escuela de pensamiento han tendido a segregar la idea de la mano invisible de Adam Smith, para plantear más terrenalmente el problema del equilibrio mercantil con base en los procesos de información.

Friedrich Hayek, uno de los líderes de la economía neoclásica, ha señalado como indudable que los seres humanos serán llevados a utilizar la mayor cantidad de información idónea, en la medida de que su remuneración depende indirectamente de circunstancias desconocidas. Es así cómo, hablando en términos de la cibernética, el mecanismo de retroalimentación (feedback) asegura el mantenimiento de un orden autogenerado que no es otra cosa que lo que antaño Adam Smith concibió como la intervención de una mano invisible. Debido a que el juego del trueque mercantil, o catalaxia, no tiene en cuenta las ideas humanas sobre lo que se debe a cada uno, y que recompen- 
sa sólo el acierto cuando se juzga sujeto a reglas formales, es como se logra la asignación más eficiente de los recursos (Hayec, 1978:51).

Ese orden se manifiesta por sí mismo, y en primera instancia, con base en que las expectativas de las transacciones se realizan con el concurso de los otros miembros de la sociedad. El ajuste mutuo de planes individuales recuerda que las ciencias físicas comenzaron años atrás a tratar con el orden espontáneo o sistema de organismos autónomos, que se llama retralimentación negativa. Esta labor la han realizado biólogos como Walter Cannon ${ }^{2}$, así como los teóricos de la comunicación y el control como Norbert Wiener, si bien, según Hayek, la idea la había trazado Adam Smith cuando hablaba de la mencionada mano invisible que regula los precios. En efecto, en un mercado libre los precios son regulados por una información o realimentación negativa (Hayec, 1991:234).

Pero no sólo la economía neoclásica apunta hacia esa dirección, sino también la economía marxista, pues como lo señaló Oscar Lange: sea Adam Smith cuando escribió sobre la mano invisible que armoniosamente dirige y coordina los procesos económicos, para acomodarlos en el estado de equilibrio. $O$ Carlos Marx cuando analizó el funcionamiento de la ley del valor como regulador de la producción de mercancías, ambos se ocuparon de los problemas de regulación de los sistemas económicos, es decir, de la cibernética (Lange, 1969:10).

Es aquí notoria una adquisición conceptual tardía por parte de la economía. Si Hayek pensó en la cibernética para explicar el funcionamiento del mercado hacia mediados de la década de 1970, sus ideas emergieron tardíamente, pues esa disciplina había sido fundada por Norbert Wiener en 1948, con la colaboración estrecha de Arturo Rosenblueth, y Julian Bigelow. Empero, Lange cree que la economía se había ocupado de antiguo de la materia de lo que ahora es la cibernética. Metafóricamente argumenta que lo que ocurre con uno de los héroes de la comedia de Moliere, monsieur Jourdain -quien se entera con sorpresa de que toda su vida habló en prosa sin saberlo-, representa muy bien la situación vivida por la economía con respecto a la cibernética. En efecto, los economistas, desde los comienzos de la economía política misma, ya se ocupaban de los problemas cibernéticos sin saberlo, pues han tratado de los procesos de ajuste y regulación de los sistemas compuestos con elementos mutuamente relacionados. Esto ocurrió incluso antes de que dichos problemas fueran planteados en otras ramas del conocimiento, como la biología, y con mucha antelación a que se formularan en forma teórica general en la más reciente ciencia de la cibernética.

2 Hayek se refiere al concepto de homeostasis formulado en 1939 por Walter Cannon, quien llamó la atención sobre la rapidez del cambio en un cuerpo cuando se alteran las condiciones donde se mueve. Se trata del intercambio con otros contextos por el cual un cuerpo no permanece inmaculado, sino sujeto a desgaste y rasgaduras, motivo por el cual desarrolla un proceso de reparación (Cannon, 1968: 256-258). 
Ciertamente, de antiguo la economía política consideraba al capitalismo como un sistema que se rige automáticamente, es decir, que opera como un sistema autorregulado. De aquí mismo surgió el principio del laissez-faire, según el cual si el Estado interviene en el curso de los procesos económicos perjudica su autorregulación y arruina el equilibrio económico que surge automáticamente. Por lo tanto, los conceptos de regulación, estabilidad, control y dirección, que pertenecen al lenguaje de la cibernética, aparecen en la literatura económica con anterioridad a que fueran objeto de investigación en cibernética.

De conformidad con lo anterior, es menester enfatizar que el mercado no es concebido como un proceso caótico sino como un gobierno, lo cual es una gran paradoja. El hecho de que se escogiera el término cibernética para referir los procesos funcionales del mercado, con base en la retroalimentación, dan finalmente la razón, al menos en parte, a quienes razonan que el gobierno es un fenómeno inevitable en casi todo acontecer humano.

\subsection{Perspectiva del Estudio de la Administración Pública}

Hoy en día la nueva gerencia públi$\mathrm{ca}$, que en su origen se concibió como un nexo entre el Estado y la sociedad a través del mercado, ha tendido a centrarse más y más en los problemas internos de la administración pública. Es decir, su temario tiende, gradualmente, a ser asimilado a los problemas clásicos de la teoría de la administración pública.

Se ha dicho, con razón, que la nueva gerencia pública es un vino viejo en nueva botella.
Quizá el más grande cambio en el nuevo manejo público globalizador consista en que, siendo originalmente un enfoque de "dentro hacia afuera" que enfatizaba esa interfase entre Estado, sociedad y mercado, ahora se está reorientando de "fuera hacia adentro". Ha dejado de soslayar el reconocimiento de que los problemas centrales en la administración pública clásica consisten en el estudio de las organizaciones del servicio público, desplazándose hacia los problemas de gobernancia, que es un tema central en ciencia política y administración pública.

Esta tendencia estaría asimilando a la neogerencia pública como un campo más de la administración pública, con la cual compartiría sus temas centrales, disipándose de tal modo el objeto de estudio y su razón de ser. Dejaría de ser, brevemente hablando, la opción a la administración pública, y la medicina para sus males, tal como se está proponiendo todavía.

\section{La Utopía Neogerencial}

La nueva gerencia pública constituye una utopía ... una utopía en el sentido que al término da Herbert Marcuse: "utopía es todo cambio en un sistema de vida que se pretende realizar dentro y conviviendo con el sistema establecido, contando sólo con la aprobación de conciencias iguales a las de sus creadores" (Sánchez-Casas y Guerra, 1973:7).

En efecto, la nueva gerencia pública se ha propuesto realizar cambios dentro del sistema a través de los cuales no se reemplace sus elementos esenciales, sino solamente se les desplace. Así, la burocracia existente pervive para dar vida al modelo posburocrático, burocra- 
cia que coexiste sin discriminaciones con el gobierno empresarial. Las antiguas estructuras políticas, administrativas y jurídicas no desaparecen, sino que se reforman para dar acomodo a un orden neogerencial que tiene suficiente con sólo sus militantes estar convencidos de sus virtudes.

De manera similar a la hermandad de los sansimonianos y los prosélitos de Charles Fourier, que formaron una escuela, la nueva utopía se congrega en torno de la escuela austriaca y la escuela de Virginia, profesándose en su seno verdades cuyos creyentes no dudan de ellas como válidas para un mundo reformable dentro de sus propios límites.

Un buen ejemplo de lo señalado lo ofrece la reforma administrativa estadounidense, la cual reconoció que no todas las operaciones federales deberían estar expuestas a la competencia y el mercado. En efecto, se reconoció por principio que no se podía hacer competir a las agencias reguladoras ni a las organizaciones políticas, porque la competencia produce guerras internas que entorpecen la hechura racional de las policies y de los programas gubernamentales. Así como la competencia sólo brinda buenos resultados cuando se aplica a la prestación de servicios públicos, puesto que es la única fuerza que no deja a las agencias otra alternativa para superarse; así la cooperación de las unidades gubernamentales es fundamental para el desarrollo de la hechura de la policy gubernamental (Gore, 1944:105).

Evidentemente muchas las actividades públicas pueden no ser sometidas a la competencia. Consiguientemente, también se deben exceptuar las opera- ciones de prestación de servicios que se realicen mejor en forma de monopolio, pues son organismos que operan como si fuesen empresas comerciales que se mueven en el mercado, pero con la diferencia de que no tienen competencia alguna (Gore, 1994:116).

En efecto, la reforma administrativa estadounidense no se propuso abolir al Estado administrativo, sino más bien, acomodar en su seno a la nueva gerencia pública con todos sus rasgos mercantiles y competitivos. Este es el sentido de utopía que tomamos de Marcuse.

\section{Conclusiones}

La sociedad post-industrial ha generado una agenda de problemas relativos a la administración pública, cuyos temas combinan asuntos perpetuos, y temas emergentes:

En primer lugar, la administración pública sigue definiéndose con base en el uso de la fuerza legítima, por cuanto emanación del Estado, sin mengua alguna por la introducción de mecanismos de mercado en algunas sus actividades económicas.

En segundo lugar, la administración pública, siendo inherentemente la administración del Estado, planeta su definición de lo administrativo como lo propia de su carácter de ciencia, así como lo económico lo es de la ciencia económica y lo político de la ciencia política. Paralelamente, siendo la administración pública inherentemente la administración del Estado, es propiamente pública, pues se debe hacer notar que el Estado representa esencialmente el aspecto público de la convivencia humana. 
En tercer lugar, si bien es cierto que la política ocupa un lugar prioritario en el estudio de la administración pública contemporánea, esto no significa que se soslaye la gerencia, pues, como lo afirmó un autor, la ciencia política es su madre natural, y el manejo su padre adoptivo.

En cuarto lugar se debe considerar a la globalización, que al aportar el factor "mercado", pone énfasis en la economía y en la organización del Estado en las materias hacendaria, industrial, agrícola, comercial y monetaria. La globalización es, asimismo, el locus donde emergió la reforma neogerencial.

En fin: hoy en día la nueva gerencia pública, que originalmente fue propuesta como un nexo entre el Estado y la sociedad a través del mercado, ha variado tan radicalmente su condición, que tiende marcadamente a centrarse en los problemas internos de la administración pública. De hecho es un vino viejo en nueva botella, pues siendo originalmente un enfoque de "dentro hacia afuera", ahora se está reorientando de "fuera hacia adentro". Esta tendencia ha ido asimilando a la nueva gerencia pública como un campo más de la administración pública, con la cual compartirá sus temas centrales.

\section{Referencias Bibliográficas}

Bell, Daniel (1973), El Advenimiento de la Sociedad Post-Industrial. Madrid, Alianza Editorial. 1976.

Bonnin, Charles-Jean (2004), Principios de Administración Pública. México, Fondo de Cultura Económica.

Cannon, Walter (1968), "Self-Regulation of the Body”. Buckley, Walter (ed.). Modern Systems Research for the Behavio- ral Scientist. Chicago, Aldine Publishing Company.

Drucker, Peter (1996), Su Visión sobre: la Administración, la Organización Basada en la Información, la Economía y la Sociedad. Santafé de Bogotá, Grupo Editorial Norma.

Fayol, Henri (1931), Administration Industrialle et Générale. Paris, Dunod.

Fayol, Henri (1927), L’Éveil de I'Esprit Public. Paris, Dunod Editeur.

Gawthrop, Louis (1988), "The Human Side of Public Administration". United States, Gaus Lecture.

Gelinier, Octave (1967), El Secreto de las Estructuras Competitivas. Madrid, Ediciones Tea.

Gide, Charles et Charles Rist (1913), Histoire des Doctrines Économiques. Paris, Libraire de la Société du Recueil Sirey.

Gore, Al (1994), Un Gobierno más Efectivo y Menos Costoso. México, EDAMEX.

Hayek, Friedrich (1978), Democracia, Justicia y Socialismo. México, Editorial Diana.

Hayek, Friedrich (1991), "Competition as a Dicovery Procedure". Petit, Philip. Contemporany Political Theory. New York, Macmillan Publishing Company.

Henry, Nicholas (1990), "Root and Branch: Public Administration's Travail Toward the Future". Lynn, Noami and Aaron Wildawsky (eds.). Public Administration: The State of Discipline. New Jersey, Chatham House Publishers. pp. 3-26.

Hintze, Otto (1968), Historia de las Formas Políticas. Madrid, Revista de Occidente. 1968.

Lange, Oscar (1969), Introducción a la Economía Cibernética. México, Siglo XXI Editores. 
Marx, Carlos (1968), El Capital. México, Fondo de Cultura Económica. Tres tomos.

Mises, Ludwig von (1996), The Human Action. Foundation for Economic Education.

Osborne, David and Ted Gaebler (1992), Reinventing Government: How the Entrepreneurial Spirit is Transforming the Public Sector. New York, Addison-Wesley Publishing Company, Inc.

Saint-Simon, Henri (1975), El Sistema Industrial. Madrid, Ediciones de la Revista del Trabajo.

Sánchez-Casas, Carlos y Felipe Guerra (1973), Fourier, ¿Socialista Utópico? Madrid, Biblioteca "Promoción de Pueblo".

Sheldon, Oliver (1986), La Filosofía del Management. Barcelona, Ediciones Orbis.
Siegfried, André (1965), El Alma de los Pueblos. Madrid, edit. Norte y Sur.

Waldo, Dwight (1948), The Administrative State. New York, The Ronald Press.

Waldo, Dwight (1967), The Study of Public Administration. New York, Random House.

Waldo, Dwight (1990), "A Theory of Public Administration Means in Our Time a Theory of Politics Also". Lynn, Naomi and Aaron Wildawsky (eds.). Public Administration: The State of Discipline. New Jersey, Chatham House Publishers.

White, Leonard (1926), Introduction to the Study of Public Administration. New York, The McMillan Co.

Wittfogel, Karl (1957), Oriental Despostism: A Comparative Study of Total Power. New Haven and London, Yale University Press. 